Fewer prevalence of $\mathrm{SH}$ were aortic stenosis (1.68\%), Cushing syndrome $(2.52 \%)$, hyperthyroidism $(0.84 \%)$, and hypothyroidism (3.36\%). (3) There were $66.24 \%$ of dyslipidemia, $43.78 \%$ of abnormality of glucose. Highest prevalence of dyslipidemia was hypertriglyceridemia (41.8\%). Diabetes and IGT were 28.6\%, $15.19 \%$, respectively. (4) The number of overweight and obesity with hypertriglyceridemia and diabetes were significantly higher than those of normal weight.

Conclusion SH should be filtrated in RH. Most RH complicated with Metabolic Disorders, it play an important role on therapy of RH to rectify Metabolic Disorders.

\section{e0287 THE THERAPEUTICAL EFFECT OF THE ADIPOSE DERIVED STEM CELLS}

doi:10.1136/hrt.2010.208967.287

Liu Qian, Wang Ke. Deparment of Cardiology, The First Affiliated Hospital of Dalian

Objective Ischaemic heart diseases, such as acute myocardial infarction, is the most important reason of heart failure. It has been thought that adult cardiac muscle cell is terminal differentiation and would not be regenerated. So now in the clinical, the myocardial ischaemia and other complications cannot be treated thoroughly. Stem cells have got a lot of attention because of the ability of selfrenewal and multi-directional differentiation. Compared with other stem cells, an important clinical advantage of adipose tissue derived stem cells (ADSCs) is that they can be isolated in real time in sufficient quantity with small injuries, so the adipose tissue will have a potential perspective. The objective of this research is to discuss the therapeutical effect of the ADSCs on acute myocardial infarction.

Methods Ligated the left anterior descending coronary artery of the $\mathrm{SD}$ rats to make a model of acute myocardial infarction. The transplantated cells were isolated from the groin and the scapular adipose tissue of the eGFP mice. The ADSCs were transplanted into the infarction area of the experimental group $(n=8)$ by injection but PBS with the same volume as the control group $(n=8)$. Four weeks later, the heart function was measured with echocardiography. The infarction size and the thickness of the ventricular wall were measured by using Masson trichrome stain. All data were expressed as mean \pm SEM. Differences between groups were analysed for statistical significance by the independent sample t-test using SPSS Statistics 11.5. A p-value less than 0.05 denoted a statistically significant difference.

Results Compared with the control group, there were smaller infarction size $(40.0 \pm 8.7 \%$ vs $79.3 \pm 27.1 \%, p<0.01)$, thicker wall $(564.1 \pm 148.1 \mu \mathrm{m}$ vs $189.7 \pm 70.0 \mu \mathrm{m}, \mathrm{p}<0.01)$ in the experimental group as well as the well improved echocardiographic functional parameters, LVESD: $(0.34 \pm 0.17 \mathrm{~cm}$ vs $0.49 \pm 0.25 \mathrm{~cm}, \mathrm{p}<0.01)$, LVEDD: $\quad(0.50 \pm 0.19 \mathrm{~cm}$ vs $0.61 \pm 0.27 \mathrm{~cm}, \quad p<0.05)$, LVFS: $[(31.5 \pm 1.8) \%$ vs $(19.4 \pm 1.4) \%, \mathrm{p}<0.01]$, LVEF: $[(65.6 \pm 3.6) \%$ vs $(45.3 \pm 2.8) \%, p<0.01]$.

Conclusion By injecting into the infarct cardiac muscle, the ADSCs have a positive effect to improve the reconstruction and the systolic and diastolic function in a rat model of acute myocardial infarction.

\section{e0288 ANALYSIS OF THE RATE OF ACHIEVING GOAL BLOOD PRESSURE, BLOOD SUGAR AND BLOOD LIPID IN PATIENT WITH CORONARY ARTERY DISEASE}

doi:10.1136/hrt.2010.208967.288

Li Yan-Fang, Cao Fang-Fang, Cao Xiao-Jing, Shi Shu-Tian. Department of Cardiology, Anzhen Hospital, Capital Medical University, Beijing

Objective To analysis the rates of goal blood pressure, blood glucose and blood lipid achieved in patients with coronary artery disease
(CAD) who were diagnosed by coronary arteriography and to explore the effect of risk factors in $\mathrm{CAD}$.

Methods 2916 cases with CAD were analysed retrospectively the levels of blood pressure, blood sugar and blood lipid when they were admitted to hospital, and the level of blood pressure when they discharged. According to goal values of guidelines, respectively, the effects of three risk factors on $\mathrm{CAD}$ were evaluated. By means of $130 / 80 \mathrm{~mm} \mathrm{Hg}$, the rate of goal blood pressure achieved was $28.4 \%$ $(829 / 2916)$ when they were admitted, and the rate of goal blood pressure achieved was $87.5 \%(2552 / 2916)$ when they were discharged. By means of $140 / 90 \mathrm{~mm} \mathrm{Hg}$, the rate of goal blood pressure achieved was $65.8 \%(1918 / 2916)$ when they were admitted, and the rate of goal blood pressure achieved was $90.0 \%(2624 / 2916)$ when they were discharged. The rate of goal high density lipoprotein cholesterol (HDL-C) achieved, by means of $>1.04 \mathrm{mmol} / \mathrm{l}$, was $34.7 \%(672 / 2916)$ when they were admitted to hospital. The rate of goal low density lipoprotein cholesterol (LDL-C) achieved, by means of $<2.59 \mathrm{mmol} / \mathrm{l}$, was $54.3 \%$ (531/978) in high risk patients, and by means of $<2.07 \mathrm{mmol} / \mathrm{l}$, was $17.4 \%$ (338/1938) in very high risk patients. The rates of goal total cholesterol (TC) achieved, by means of $<4.14 \mathrm{mmol} / \mathrm{l}$, was $49.5 \%$ (484/978) in high risk patients, and by means of $<3.11 \mathrm{mmol} / \mathrm{l}$, was $5.83 \%(113 / 1938)$ in very high risk patients. The rates of goal triglyceride achieved, by means of $<1.7 \mathrm{mmol} / \mathrm{l}$, was $58.0 \%(1690 / 2916)$. There was 538 cases diabetic patients in CAD, occupying $18.5 \%$ (538/2916), diabetic diagnosis standard was fasting blood glucose $>7.0 \mathrm{mmol} / \mathrm{l}$. The rates of goal fasting blood glucose was $1.86 \%(10 / 538)$, by means of $<6.0 \mathrm{mmol} / 1$ fasting blood glucose. There was 600 patients of fasting blood glucose impaired, by means of $>5.6 \mathrm{mmol} / \mathrm{l}$ and $<7.0 \mathrm{mmol} / \mathrm{l}$ on fasting blood glucose, and diabetes together with fasting blood glucose impaired were 1138 cases, occupying 39.03\% (1138/2916) in $\mathrm{CAD}$.

Conclusion The rates of goal blood pressure, fasting blood glucose and blood lipid achieved in patients with CAD were low when they were admitted. Not only rate of goal fasting blood glucose was very low in $\mathrm{CAD}$ accompanying diabetic patients, and but also rates of goal TC and LDL-C achieved were low in CAD. The results show that the management of goal blood pressure, goal fasting blood glucose and goal blood lipid are very important in prehospital for secondary prevention of CAD.

\section{e0289 ASSOCIATION OF LOW ANKLE-BRACHIAL INDEX WITH MORTALITY IN PATIENTS WITH ISCHAEMIC HEART DISEASE}

doi:10.1136/hrt.2010.208967.289

'Zheng Liqiang, ${ }^{2} \mathrm{Li}$ Jue, ${ }^{2} \mathrm{Hu}$ Dayi, ${ }^{3}$ Luo Yingyi, ${ }^{2} \mathrm{Li}$ Xiankai, Xu Yuanxi. ${ }^{1}$ Department of Cardiology, Shengjing Hospital of China Medical University, Shenyang, China; ${ }^{2}$ Heart, Lung and Blood Vessel Center, Tongji University, Shanghai, China; ${ }^{3}$ Shanghai Institute of Health Sciences, Shanghai, China

Objective To find out whether a low ankle-brachial index can improve the prediction of and cardiovascular mortality on top of conventional risk factors remains unclear among patients with ischaemic heart disease. The present study was to assess the association between ankle-brachial index and mortality in Chinese patients.

Methods An observational prospective study was conducted in which 1,800 Chinese patients aged $\geq 35$ years were followed-up from 2004 to 2007-2008.

Results There were 280 deaths, of which 165 were attributable to cardiovascular disease. Compared with patients with an anklebrachial index $\geq 1.1$, the risk of mortality increased linearly in lower ankle-brachial index categories: patients with an ankle-brachial index of 0.9 to $1.1,0.7$ to $0.9,<0.7$ had HR of $1.60,2.07,3.08$ for mortality and 1.89, 2.33, 4.09 for cardiovascular mortality ( $p$ for 
trend $<0.001)$ respectively. Addition of ankle-brachial index significantly $(p<0.001)$ increased the predictive value of the model for 3 year deaths compared with a model containing risk factors alone. Comparison of areas under receiver operator characteristics curves confirmed that a model including the ankle-brachial index discriminated better than one without.

Conclusions There was an inverse association between anklebrachial index and mortality. Addition of ankle-brachial index significantly improved the prediction of 3-year mortality over and above that of conventional risk factors. We recommend that anklebrachial index be incorporated into prognostic assessment for patients with ischaemic heart disease.

\section{e0290 LOWER ESTIMATED GLOMERULAR FILTRATION RATE CORRELATES TO ARTERIAL STIFFNESS IN GENERAL CHINESE WOMEN WITH NORMAL RENAL FUNCTION}

doi:10.1136/hrt.2010.208967.290

Bian Suyan, Luo Leiming, Xiao Tiehui, Oi Liping, Wu Hongmei, Xiao Wenkai, Sheng Li, Duan Liufa. Chinese Pla General Hospital

Background and Objective Although extensive research has shown the association of decreased renal function with early arteriosclerosis in patients with chronic kidney disease, it's still controversy whether decreased glomerular filtration rate (GFR) and menopause have independent relationship with arterial stiffness in general women residents with normal renal function. The aim of this study was to identify how decreased GFR or menopause contributes to central and peripheral arterial stiffness.

Methods In this cross-sectional study, we randomly recruited 1,131 women residents (mean age: $56.59 \pm 14.16$ years) with normal GFR levels estimated by the abbreviated Modification of Diet in Renal Disease equation (defined as eGFR $\geq 60 \mathrm{ml} / \mathrm{min} / 1.73 \mathrm{~m}^{2}$ ) from three large communities in Beijing. Arterial stiffness was assessed by measuring carotid-femoral PWV (PWVcf), carotid-radial PWV (PWVcr) and augmentation index (AIx) with validated automatic devices. Menopause status was defined by the cut-off age of 50, the median menopause age of Chinese women.

Results Subjects from the lowest eGFR tertile group had the highest PWVcf and AIx, while the values of PWVcr were comparable. Meanwhile, eGFR was inversely correlated with PWVcf and AIx but not with PWVcr. Multiple stepwise regression demonstrated a significant relationship between eGFR and PWVcf, independent of the conventional atherosclerotic risk factors. This association was not significant between eGFR values and PWVcr or AIx. Lower eGFR accompanied by higher PWVcf and AIx was evident in the post-menopause group while the PWVcr values were comparable. Post-menopause was an independent predictor for PWVcf and AIx, but not for PWVcr.

Conclusions In general Chinese women with normal renal function, decreased eGFR seems to affect the core arteries other than the peripheral ones. Post-menopause might play part role in arterial stiffness.

\section{e0291 EFFECTS OF XINFUKANG ORAL LIQUID ON THE ACTIVITIES OF RESPIRATORY ENZYME IN EXPERIMENTAL CONGESTIVE HEART FAILURE RATS}

doi:10.1136/hrt.2010.208967.291

Weiwe Wang, Xuebin Cao, Shule Xu, Jiancheng He, Yuanhui Hu. Department of Cardiology, 252 Hospital of PLA

Objective To test the effect of Xin Fu Kang Oral Liquid on the activities of respiratory enzyme (I-IV) in pressure overload-induced left ventricular hypertrophy in rats.
Methods The models of congestive heart failure (CHF) were established by constricting the abdominal aorta of rats partly. $75 \mathrm{SD}$ rats were randomly divided into Sham operation $(\mathrm{SH})$, Coarctation of abdominal aorta model group (CAA) and Xinfufang Oral Liquid group (XFK). The activities of respiratory enzyme (I-IV) were respectively measured by spectrophotometric method in every group at the 10th, 12th week after the interventional of the drugs. Results The study shows that CAA group the activities of respiratory enzyme significantly decreased, the activities of respiratory enzyme II (SDH), IV (CCO) have obviously difference ( $p<0.01)$, In the XFK group the activities of respiratory enzyme obviously increased compared with CAA and by the 10th, 12thweek, SDH, CCO have obviously difference $(p<0.01)$, The activities of respiratory enzyme of the 12th week in XFK group obviously increased compared with that of the 10th week, SDH, CCO have obviously difference $(p<0.05)$.

Conclusion XinFuKang Oral Liquid can obviously improve the activities of respiratory enzyme of congestive heart failure rats.

\section{E0292 SURVEY OF CURRENT SMOKING STATUS OF PATIENTS AT CARDIOLOGY CLINICS IN BEIJING}

doi:10.1136/hrt.2010.208967.292

${ }^{1}$ Rong Jing Ding, ${ }^{2}$ Jinming Yu, ${ }^{2}$ Lijun Zhang, 'Dayi Hu. ${ }^{1}$ Heart and Vascular Center,

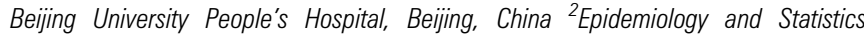
department, Fudan University, ShangHai, China

Objective To understand the current status of smoking patients at cardiology clinics in Beijng municipal Class 3A hospital, so as to target the anti-smoking intervention.

Methods During March 1-10, 2009, the patients presenting at cardiology clinics were consecutively recruited and a questionnaire survey conducted.

Results A total of 816 valid questionnaires were collected. There were 531 males and 336 females with an age range of 22-80 years old. Among these patients, $90.5 \%$ knew the fact that smoking was harmful to cardiovascular health, $67.4 \%$ non-smokers, $15.8 \%$ smokers and $15.8 \%$ quitters. The smoking rate among the patients with coronary heart disease, diabetes mellitus, hyperlipidaemia and hypertension was $28.4 \%, 46.7 \%, 50 \%$ and $73.3 \%$ respectively. The smokers consumed a median of 15 cigarettes per day. $71.8 \%$ of them did not wish to quit. Those with nicotine dependence score under 4 accounted for $64.2 \%$ and above 5 accounted for $35.8 \%$. The quitting urge had no correlation with nicotine dependence score or cardiovascular disease categories.

Conclusion The smoking rate among the patients presenting at cardiology clinic is lower than that of general population. The smokers had a lesser urge to quit. Besides strengthening smokingquitting interventions in coronary heart disease patients an outpatient department, cardiologist should also do so for those smokers with cardiovascular risk factors.

\section{E0293 SURVEY 2009 OF CURRENT SMOKING STATUS OF URBAN AND RURAL RESIDENTS IN BEIJING REGION}

doi:10.1136/hrt.2010.208967.293

${ }^{1}$ Rong Jing Ding, ${ }^{2}$ Jinming Yu, ${ }^{2}$ Lijun Zhang, ${ }^{1}$ Dayi Hu. ${ }^{1}$ Heart and Vascular Center, Beijing University People's Hospital, Beijing, China ${ }^{2} E$ Eidemiology and Statistics Department, Fudan University, ShangHai, China

Objective To understand the smoking rate, distribution characteristics and quitting urge of urban and rural populations in Beijing region in 2009 so as to provide basal materials for future antismoking population interventions. 